\begin{tabular}{|c|c|}
\hline Title & Detection of discrete surface charge dy namics in GaA s based nanowire through metal-tip-induced current fluctuation \\
\hline Author(s) & Sato, Masaki; Y in, Xiang; Kuroda, Ryota; Kasai, Seiya \\
\hline Citation & $\begin{array}{l}\text { Japanese Journal of A pplied Physics(JJA P), 55(2S), 02BD } 01 \\
\text { https://doi.org/10.7567/JAA P.55.02BD01 }\end{array}$ \\
\hline Issue Date & 2016-02 \\
\hline Doc URL & http:/hdl.handle.net/2115/66630 \\
\hline Rights & (c) 2016 The Japan Society of A pplied Physics \\
\hline Type & article (author version) \\
\hline File Information & JJA P2015_55_EM15049.pdf \\
\hline
\end{tabular}

Instructions for use 


\title{
Detection of discrete surface charge dynamics in GaAs-based nanowire through metal-tip-induced current fluctuation
}

\author{
Masaki Sato*, Xiang Yin, Ryota Kuroda, and Seiya Kasai*
}

Research Center for Integrated Quantum Electronics and Graduate School of Information Science \& Technology, Hokkaido University, Sapporo 060-8628, Japan

*e-mail: msato@rciqe.hokudai.ac.jp, kasai@rciqe.hokudai.ac.jp

We investigated the detection of discrete charge dynamics of an electron trap in a GaAs-based nanowire surface through current fluctuation induced by a metallic scanning probe tip. An equivalent circuit model indicated that the charge state in the surface strongly reflects the channel potential when the local surface potential is fixed by the metal tip, which suggests that random charging and discharging dynamics of the trap appears as random telegraph signal (RTS) noise in the nanowire current. Experimental demonstration of the concept was carried out using a GaAs-based nanowire and an atomic force microscope (AFM) system with a conductive tip. We observed the RTS noise in the drain current and superposition of the Lorentzian component in the noise spectrum when the metal tip was in contact with the nanowire surface at specific positions. The obtained results indicate the possibility of detecting charge dynamics of the individual surface trap in semiconductor devices. 


\section{Introduction}

Electron traps in a semiconductor surface significantly influence the electrical characteristics of semiconductor devices. Random charging and discharging of the trap causes temporal fluctuation of the electrostatic potential in the device, which often appears as current or voltage noise and often affects various device performances. Because fluctuation becomes obvious as the size of the device decreases ${ }^{1-3)}$, the characterization and understanding of the charge dynamics of a surface trap has became an important issue in recent years. Although there are various scanning probe techniques that characterize the surface at an atomic scale, such as scanning tunneling spectroscopy (STS) ${ }^{4-6)}$ and electrostatic force microscopy $(\mathrm{EFM})^{7-11)}$, they mostly characterize static properties of the surface. The detection of the dynamics of the electric properties is difficult for such systems because the small conductance of the tiny tip contact results in a delay of the electric response. Here, we focus on scanning gate microscopy (SGM) for characterizing the local transport properties of electron devices ${ }^{12-24)}$. In this technique, the biased conducting tip of an atomic force microscope (AFM) modulates electrostatic potential locally in the device and the current passing through the device, which is not influenced by the tip conductance, is measured. In this paper, we propose and demonstrate the detection of discrete charge dynamics of electron traps in the semiconductor surface through the measurement of current noise induced by contacting a metal tip to the surface. First we describe our concept of detecting charge dynamics in the semiconductor surface. Next the proposed technique is demonstrated using an AFM system with a metal tip. Then we discuss possible approaches to obtaining information on individual traps from the measured noise.

\section{Concept}

The basic concept of detecting discrete charge dynamics of a trap in the semiconductor surface is shown in Fig. 1. Here, the surface of a semiconductor nanowire is characterized A metal tip comes into contact with the surface of the semiconductor nanowire channel 
having source and drain electrodes. A biased conducting tip acts as a small movable gate that modulates the electrostatic potential locally in the device. Drain voltage $V_{\mathrm{D}}$ is applied to the channel and drain current $I_{\mathrm{D}}$ is measured in the time domain. When a trap exists in the nanowire surface, it stochastically captures and emits an electron owing to thermal fluctuation. This process modulates the channel potential and is expected to cause drain current noise having binary states, called random telegraph signal (RTS) noise. We recently found that the metal contact on the surface enhanced such current noise, from the analysis on the current noise in a GaAs-based nanowire FET with single-molecule dispersion $^{25)}$. Figures 1(b) and 1(c) schematically show the sample without and with a metal tip contact, respectively, together with corresponding equivalent circuits. $C_{1}$ is the capacitance between the tip and the trap and $C_{2}$ is the tunnel capacitance between the trap and the channel, respectively. The trap is represented by a node between capacitors $C_{1}$ and $C_{2}$. Charging and discharging of the trap is assumed to occur through $C_{2}$. When the tip is retracted, the drain current will show small noise, as shown in Fig. 1(b). In this case, from the analysis of the equivalent circuit, the channel potential $V_{\mathrm{C}}$ is found to be independent of the charge in the trap $e$,

$$
V_{C}=Q / C_{3}
$$

where $Q$ is the charge of the electron channel and $C_{3}$ is the capacitance of the GaAs substrate. On the other hand, when the tip touches the surface over the electron trap, the tip capacitively couples with the trap, as shown in Fig. 1(c), and the charge in the trap, in turn, influences the electrostatic potential inside the device. Computing the equivalent circuit, the channel potential is given by

$$
V_{C}=\frac{\left(C_{1}+C_{2}\right) Q+C_{2} e}{C_{1} C_{2}+C_{2} C_{3}+C_{3} C_{1}} .
$$

It is found that $V_{\mathrm{C}}$ depends on $e$. Therefore, as shown in Figs. 1(b) and 1(c), RTS noise appears when a metal tip comes into contact with the surface over a trap, whereas it disappears when the tip is retracted. If there is no trap underneath the tip, RTS noise will 
not appear even when the tip is in contact with the surface. On the basis of this mechanism, we can detect a discrete trap in the semiconductor surface by observing RTS noise induced by a very small metal tip.

In this study, we characterize the trap in the surface of a III-V compound semiconductor nanowire device, since the nanowire current is very sensitive to the surface charge owing to the high surface-area-to-volume ratio and high electron mobility. In an actual case, current in the device often includes $1 / f$ noise ${ }^{26-29)}$, which is generated by a large number of traps distributed uniformly relative to the time constant ${ }^{30)}$. Although RTS noise caused by the above mechanism might be small and difficult to distinguish from other noise, it shows a Lorentzian spectrum of $1 / f^{2}$ slope $^{31,32)}$. When the discrete charge dynamics of a trap takes place, the Lorentzian spectrum will be superimposed onto the $1 / f$ noise spectrum, which can be detected using a spectrum analyzer having a wide dynamic range and can be distinguished from other noise by an appropriate spectrum decomposition technique.

\section{Experimental procedure}

To demonstrate the above concept, we prepared the system shown in Fig. 1(a) using an atomic force microscopy (AFM) system. An etched GaAs-based nanowire was used for the device under test. The conductive AFM tip was covered with PtIr. The radius of the tip was $30 \mathrm{~nm}$. In this system, the current in the nanowire was simultaneously measured with a contacting tip. The GaAs-based nanowire was fabricated on the modulation-doped $\mathrm{AlGaAs} / \mathrm{GaAs}$ heterostructure having two-dimensional electron gas (2DEG) by electron beam lithography and wet chemical etching using a $\mathrm{H}_{2} \mathrm{SO}_{4}$-based etchant. The heterostructure consisted of a $10 \mathrm{~nm} \mathrm{n}^{+}$-GaAs cap layer, $50 \mathrm{~nm}$ undoped AlGaAs top barrier, Si delta-doping layer, $12 \mathrm{~nm}$ undoped AlGaAs spacer, $20 \mathrm{~nm}$ undoped GaAs channel, 100-nm AlGaAs bottom barrier, and undoped GaAs buffer on a semi-insulating (001) GaAs substrate. The sheet density of the 2DEG was $7.8 \times 10^{11} \mathrm{~cm}^{-2}$ and the mobility was $7,100 \mathrm{~cm}^{2} \mathrm{~V}^{-1} \mathrm{~s}^{-1}$ at $300 \mathrm{~K}$. The length and width of the fabricated nanowire were $10 \mu \mathrm{m}$ and $800 \mathrm{~nm}$ respectively. The direction of the nanowire was $<\overline{1} 10>$. Ni/Ge/Au/Ni/Au ohmic contacts were formed for the source and drain electrodes. The drain current was 
once amplified and converted to voltage using a low-noise amplifier whose amplification gain was $10 \mathrm{kV} / \mathrm{A}$. We characterized the drain current in the nanowire using a voltage monitor or a spectrum analyzer. Drain voltage $V_{\mathrm{D}}$ was set at $0.85 \mathrm{~V}$, where the current noise in the fabricated device sufficiently exceeded the noise floor of the measurement system of $10^{-23} \mathrm{~A}^{2} / \mathrm{Hz}$. The AFM tip was connected to the ground and its potential was kept at zero in this study. All the measurements were carried out at room temperature.

\section{Results}

\subsection{Current noise in time domain}

Figure 2 shows measured drain currents with tip contact at three different surface positions, together with an AFM image of the fabricated sample. When the tip was located at position 1, the drain current showed small noise, as shown in Fig. 2(a). However, when the tip was located at positions 2 and 3, RTS noise was clearly superimposed, as shown in Figs. 2(b) and 2(c). The obtained results suggested that the traps existed in the surface region under positions 2 and 3. These RTS noise waveforms had different intensities and time constants. Such differences indicated that we detected different discrete traps. The RTS waveform at position 2 provided a charging time constant $\tau_{\mathrm{c}}$ of $0.86 \mathrm{~ms}$ and discharging time constant $\tau_{\mathrm{d}}$ of $2.3 \mathrm{~ms}$. Here $\tau_{\mathrm{c}}$ and $\tau_{\mathrm{d}}$ are defined as shown in Fig. 1(c). We also evaluated the average time constant $\tau_{0}$ from $\tau_{\mathrm{c}} \tau_{\mathrm{d}} /\left(\tau_{\mathrm{c}}+\tau_{\mathrm{d}}\right)$ and $\tau_{0}=0.63 \mathrm{~ms}$ was obtained. Similarly, the charging and discharging time constants for the RTS at position 3 were $\tau_{\mathrm{c}}=234 \mathrm{~ms}$ and $\tau_{\mathrm{d}}=578 \mathrm{~ms}$, respectively, and $\tau_{0}=167 \mathrm{~ms}$ was obtained.

Figure 3 shows the tip position dependence of noise intensity at $V_{\mathrm{D}}=0.85 \mathrm{~V}$. The noise intensity was measured as the variance of the measured noise amplitude when the tip was in contact with the surface. The interval between measured positions was approximately $1 \mu \mathrm{m}$. The intensity of noise with the tip contact depended on the tip position. The noise intensity increased as the tip position became close to the source electrode, as shown in Fig. 3. Such dependence disappeared when the tip was retracted.

Figure 4 shows histograms of the drain current with the tip contact at positions 2 and 3. The histograms exhibited two clear peaks corresponding to binary states in the potential inside. This again indicated discrete charge dynamics. The intervals of the current 
peaks in Figs. 4(a) and 4(b) were 26 and $61 \mathrm{nA}$, respectively, which were equal to the average amplitudes of the RTS in Fig. 2. The obtained histograms also showed different peak intensities between the two peaks. The ratio of the two peak intensities corresponds to the ratio of the charging and discharging time constants, $\tau_{\mathrm{c}} / \tau_{\mathrm{d}}$, which was evaluated to be 0.37 and 0.4 for the RTS with tip contacts at positions 2 and 3, respectively. The values were in reasonable agreement with the ratios from the time constants directly evaluated from the RTS waveforms in Fig. 2. The differences in the amplitudes of RTS and the ratios of the charging and discharging time constants of RTS are expected to include physical information on the trap.

\subsection{Current noise spectra}

Figure 5 shows measured drain current noise spectra. When the tip was retracted from the surface, the $1 / f$ noise was dominant. When the tip was brought into contact with the surface at position 1, a similar 1/f noise was again observed, as shown in Fig. 5(a). The noise power was also the same. On the other hand, when the tip came into contact with the surface at positions 2 and 3 , the noise was increased and the configuration of the spectrum was changed. The results indicated that the tip affected the noise spectrum only when a trap existed underneath the tip. The spectrum could be well fitted by the combination of $1 / f$ and Lorentzian components,

$$
S_{I_{D}}=a \frac{1}{f}+b \frac{1}{1+\left(f / f_{0}\right)^{2}}
$$

where $f_{0}$ is a corner frequency of the Lorentzian spectrum and $a, b$, and $f_{0}$ are fitting parameters. From the fitting, corner frequencies of $f_{0}=580$ and $1.8 \mathrm{~Hz}$ were obtained for RTS noise at positions 2 and 3, respectively. The relationship between the corner frequency and the average time constant $\tau_{0}$ is given by $2 \pi f_{0}=1 / \tau_{0}$. The estimated $f_{0}$ from $\tau_{0}$ $=0.63 \mathrm{~ms}$ in RTS at position 2 was $253 \mathrm{~Hz}$, which was in reasonably good agreement with the corner frequency of $580 \mathrm{~Hz}$ from the noise spectrum. Similarly, $\tau_{0}=167 \mathrm{~ms}$ from RTS at position 3 corresponded to $f_{0}=0.95 \mathrm{~Hz}$, which was again reasonably in agreement with the $f_{0}=1.8 \mathrm{~Hz}$ from the noise spectrum at position 3 . Therefore, the origin of the 
superimposed Lorentzian spectrum at positions 2 and 3 was the same to that for the generation of the RTS in Fig. 2. The difference in the corner frequency was reflected in the information of the trap energy and the depth position.

\section{Discussion}

First we verify that the observed RTS arose from the charge dynamics of a single trap. For verification, we estimated the possible current change $\Delta I_{\mathrm{D}}$ owing to charging and discharging of an electron in a trap. In the case of the field-effect transistor (FET), $\Delta I_{\mathrm{D}}$ is given by $g_{\mathrm{m}} e / C_{\mathrm{G}}$, where $g_{\mathrm{m}}$ is the transconductance and $C_{\mathrm{G}}$ is the gate capacitance of the $\mathrm{FET}^{31)}$. The value of $e / C_{\mathrm{G}}$ corresponds to the effective gate voltage $\Delta V_{\mathrm{G}}$ induced by charging or discharging an electron to the gate capacitance. In our case, effective gate voltage is estimated from eq. (2) instead of $e / C_{\mathrm{G}}$. Then $\Delta I_{\mathrm{D}}$ is given by

$$
\Delta I_{D}=g_{m} \frac{C_{2} e}{C_{1} C_{2}+C_{2} C_{3}+C_{3} C_{1}},
$$

where $C_{1}=\varepsilon_{\mathrm{AlGaAs}} S / d_{1}, C_{2}=\varepsilon_{\mathrm{AlGaAs}} S / d_{2}, C_{3}=\varepsilon_{\mathrm{GaAs}} \mathrm{S} / d_{3}, \varepsilon_{\mathrm{AlGaAs}}$ is the permittivity of AlGaAs, $\varepsilon_{\mathrm{GaAs}}$ is the permittivity of GaAs, $d_{1}$ is the depth of the surface trap from the surface, $d_{2}$ is the distance between the surface trap and the channel, and $d_{3}$ is a thickness of the layer under the 2DEG channel. The effective tip area in contact with the surface was estimated to be $2.6 \times 10^{3} \mathrm{~nm}^{2}$ from the tip geometry $\frac{33)}{}$. We estimated $g_{\mathrm{m}}$ of $18.8 \mathrm{mS} / \mathrm{mm}$ from the material parameters and the device geometry. Because the depth position of the trap was unknown, we calculated $\Delta I_{\mathrm{D}}$ and plot the result in Fig. 6 as a function of $d_{1}$. The calculated $\Delta I_{\mathrm{D}}$ varies in the range of 0 to $80 \mathrm{nA}$ depending on the depth position of the trap. The experimentally observed $\Delta I_{\mathrm{D}}$ of 26 and $61 \mathrm{nA}$ for tip contact at positions 2 and 3 can be explained by assuming $d_{1}=21 \mathrm{~nm}$ and $49 \mathrm{~nm}$, respectively. Although the depth position of the trap was not just at the surface at an atomic level, it was reasonable to consider that the observed RTS was caused by charging and discharging of a single trap between the surface and the channel. To clarify the depth position precisely, further analysis is necessary. Charging and discharging time constants also include information on the depth 
position. A thick barrier between the channel and the trap should prolong the charging and discharging time constants even with the same $\tau_{\mathrm{c}} / \tau_{\mathrm{d}}$.

The observed tip-position dependence of the RTS intensity in Fig. 3 may indicate that the influence of the tip contact is significant on the source side. However, we considered this to be unlikely. In the case of the GaAs-based nanowire, drain voltage induces a larger electric field and depletes the channel on the drain side of the nanowire owing to strong surface Fermi level pinning ${ }^{34)}$, which means that the potential on the source side is rather flat and no abrupt change in the capacitive couplings between the tip, trap, and channel is expected. This point will be verified by the same measurement with changing source and drain electrodes or by the measurement of other samples. In addition, the surface trap density of the GaAs-based material is often as high as $10^{12} \mathrm{~cm}^{-2}$, indicating that traps exist at approximately $10 \mathrm{~nm}$ intervals. To obtain the actual spatial distribution of the individual traps, it is necessary to measure current by changing the tip position with intervals of less than $10 \mathrm{~nm}$. Such measurements are the aims of our future work.

Finally we discuss the energy level of the trap. Information on the trap energy $E_{\mathrm{T}}$ can be obtained from

$$
\frac{\tau_{c}}{\tau_{d}}=\exp \left(-\frac{E_{T}-E_{F}}{k T}\right),
$$

where $E_{\mathrm{F}}$ is the Fermi energy, $k$ is the Boltzmann constant, and $T$ is the temperature. The evaluated $E_{\mathrm{T}}-E_{\mathrm{F}}$ of the traps at positions 2 and 3 were -26 and $-24 \mathrm{meV}$, respectively. Almost the same energy was obtained for the two positions, since the values of $\tau_{\mathrm{c}} / \tau_{\mathrm{d}}$ were similar to each other. The evaluated trap energy was rather shallow compared with the well-known deep trap in AlGaAs, that is, DX center with an activation energy of around $0.4 \mathrm{eV}^{35)}$. Considering the band bending in the $\mathrm{AlGaAs} / \mathrm{GaAs}$ modulation doped structure with a large surface potential of around $0.8 \mathrm{eV}$ in $\mathrm{GaAs}^{36)}$, the trap energy could be aligned to the Fermi level of the 2DEG channel when the trap depth position is around $25 \mathrm{~nm}$ from the surface. Bringing a metal tip into contact with the surface also affected the surface potential, which would influence the trap energy estimation. To obtain the further information of the trap energy, it will be useful to characterize the tip-bias dependence of 
the charging and discharging time constants. If the tip bias can achieve the flat band condition, activation energy from conduction band will be evaluated using Eq. (5). The bias dependence of the time constants should change depending on the trap depth position, which also provides information on depth position. Such comprehensive analysis of the RTS time constants, amplitude, and their tip bias dependence in our technique will reveal the physical properties of the individual trap. This remains as our future work.

\section{Conclusions}

We proposed and demonstrated a novel detection technique of discrete charge dynamics of a trap in a GaAs-based nanowire surface using current fluctuation induced by a metal tip. A simple equivalent circuit model indicated that the random telegraph signal (RTS) noise was imposed in the drain current when the metal tip came into contact with the surface. The concept was successfully demonstrated by measuring current noise in a GaAs-based nanowire set in an atomic force microscope (AFM) system with a metal tip, where RTS noise with a Lorentzian spectrum was superimposed when the metal tip came into contact with the nanowire surface at specific positions. From the intensity and time constant of the RTS noise, we could obtain information on the energy and position of the discrete trap. The obtained results showed the possibility of detecting charge dynamics of the individual surface traps in semiconductor devices.

\section{Acknowledgment}

This work was partly supported by a Grant-in-Aid for Scientific Research on Innovative Areas "Molecular Architectonics" (No. 25110001) from the Ministry of Education, Culture, Sports, Science and Technology, Japan. 


\section{References}

1) F. N. Hooge, IEEE Trans. Electron Devices 41, 1926 (1994).

2) H. Ueno, T. Kitamura, S. Matsumoto, T. Okagaki, M. Miura-Mattausch, H. Abe, and T. Hamasaki, Appl. Phys. Lett. 78, 380 (2001).

3) T. Ohmi, M. Hirayama, and A. Teramoto, J. Phys. D 39, R1 (2006).

4) H. Hasegawa, N. Negoro, S. Kasai, Y. Ishikawa, and H. Fujikura, J. Vac. Sci. Technol. B 18, 2100 (2000).

5) S. Kasai, N. Negoro, and H. Hasegawa, Appl. Surf. Sci. 175-176, 255 (2001).

6) N. Negoro, S. Kasai, and H. Hasegawa, Appl. Surf. Sci. 269-274, 190 (2002).

7) E. Bussman, D. Jun Kim, and C. C. Williams, Appl. Phys. Lett. 85, 2538 (2004).

8) E. Bussman, N. Zheng, and C. C. Williams, Appl. Phys. Lett. 86, 163109 (2005).

9) E. Bussman and C. C. Williams, Appl. Phys. Lett. 88, 263108 (2006).

10) J. P. Johnson, D. W. Winslow, and C. C. Williams, Appl. Phys. Lett. 98, 052902 (2011).

11) D. Winslow and C. Williams, J. Appl. Phys. 110, 114102 (2011).

12) M. A. Topinka B. J. LeRoy, S. E. J. Shaw, E. J. Heller, R. M. Westervelt, K. D. Maranowski, and A. C. Gossard, Science 289, 2323 (2000).

13) M. A. Topinka B. J. LeRoy, R. M. Westervelt, S. E. J. Shaw, R. Fleischmann, E. J. Heller, K. D. Maranowski, and A. C. Gossard, Nature 410, 183 (2001).

14) M. P. Jura, M. A. Topinka, M. Grobis, L. N. Pfeiffer, K. W. West, and D. Goldhaber-Gordon, Phys. Rev. B 80, 041303 (2009).

15) N. Aoki, C. R. Da Cunha, R. Akis, D. K. Ferry, and Y. Ochiai, Appl. Phys. Lett. 87, $223501(2005)$.

16) N. Aoki, C. R. da Cunha, R. Akis, D. K. Ferry, and Y. Ochiai, Phys. Rev. B 72, 155327 (2005).

17) N. Aoki, K. Sudou, K. Okamoto, J. P. Bird, and Y. Ochiai, Appl. Phys. Lett. 91, 192113 (2007).

18) N. Aoki, R. Brunner, A. M. Burke, R. Akis, R. Meisels, D. K. Ferry, and Y. Ochiai, Phys. Rev. Lett. 108, 136804 (2012).

19) F. Martins B. Hackens, M. G. Pala, T. Ouisse, H. Sellier, X. Wallart, S. Bollaert, A. Cappy, J. Chevrier, V. Bayot, and S. Huant, Phys. Rev. Lett. 99, 136807 (2007). 
20) J. Liu, Z. Cai, and G. Koley, J. Appl. Phys. 106, 124907 (2009).

21) A. A. Zhukov, Ch. Volk, A. Winden, H. Hardtdegen, and Th. Schäpers, JETP Lett. 93, 10 (2011).

22) A. A. Zhukov, Ch. Volk, A. Winden, H. Hardtdegen, and Th. Schäpers, J. Exp. Theor. Phys. 116, 138 (2013).

23) D. Martin, A. Heinzig, M. Grube, L. Geelhaar, T. Mikolajick, H. Riechert, and W. M. Weber, Phys. Rev. Lett. 107, (2011) 216807.

24) J. L. Webb, O. Persson, K. A. Dick, C. Thelander, R. Timm, and A. Mikkelsen, Nano Res. 7, 877 (2014).

25) S. Inoue, R. Kuroda, X. Yin, M. Sato, and S. Kasai, Jpn. J. Appl. Phys. 54, 04DN07 (2015).

26) K. Miura, Y. Shiratori, and S. Kasai, Jpn. J. Appl. Phys. 50, 06GF18 (2011).

27) T. Muramatsu, K. Miura, Y. Shiratori, Z. Yatabe, and S. Kasai, Jpn. J. Appl. Phys. 51, 06FE18 (2012).

28) P. Viktrovitch, P. Rojo-Romeo, J. L. Leclercq, X. Letartre, J. Tardy, and M. Gendry, IEEE Trans. Electron Devices 43, 2085 (1996).

29) Y.-J. Chan and D. Pavlidis, IEEE Trans. Electron Devices 41, 637 (1994).

30) A. G. Jordan and N. A. Jordan, IEEE Trans. Electron Devices 12, 148 (1965).

31) N. Clément, K. Nishiguchi, A. Fujiwara, and D. Vuillaume, Nat. Commun. 1, 92 (2010).

32) L. K. J. Vandamme and F. N. Hooge, IEEE Trans. Electron Devices 55, 3070 (2008).

33) S. Hudlet, M. Saint Jean, C. Guthmann, and J. Berger, Eur. Phys. J. B 2, 5 (1998).

34) M. Sato and S. Kasai, Jpn. J. Appl. Phys. 52, 06GE08 (2013).

35) P. M. Mooney, J. Appl. Phys. 67, R1 (1990).

36) H. Hasegawa and H. Ohno, J. Vac. Sci. Technol. B 4, 1130 (1986). 


\section{Figure Captions}

Fig. 1. (Color online) Concept of detection of charge dynamics of trap in semiconductor surface. (a) Detection setup including AFM system with a metal tip together with $I_{\mathrm{D}}-V_{\mathrm{D}}$ measurement system for GaAs-based nanowire sample. (b, c) Equivalent circuits of the system without and with metal tip contact on the sample, respectively. Schematic views of the sample are also shown.

Fig. 2. (Color online) Measured drain currents with metal tip contact at three different surface positions together with AFM image of the fabricated sample: (a) position 1 , (b) position 2, and (c) position 3 .

Fig. 3. (Color online) Drain current noise intensity as a function of lateral tip contact position along the nanowire direction.

Fig. 4. (Color online) Histograms of drain current noise with metal tip contact at (a) position 2 and (b) position 3.

Fig. 5. (Color online) Measured noise spectra with tip contact at (a) position 2 and (b) position 3. Noise spectrum at which tip was retracted is also shown.

Fig. 6. (Color online) (a) Schematic view and equivalent circuit of the sample including a trap and (b) calculated RTS amplitude $\Delta I_{\mathrm{D}}$ as a function of depth position of a trap $d_{1}$. 


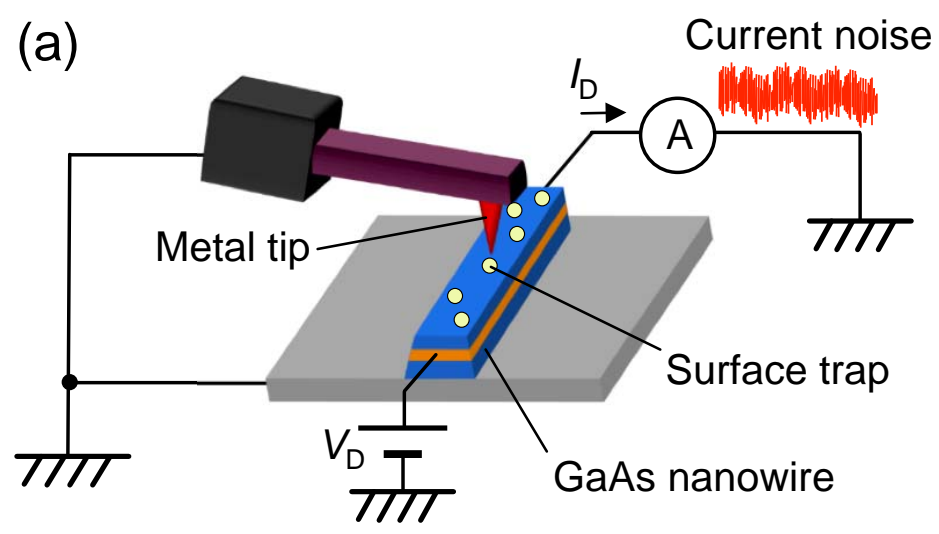

(b)
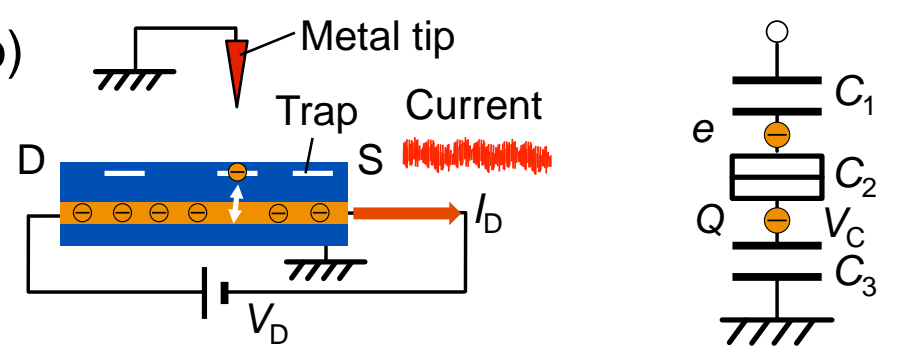

(c)

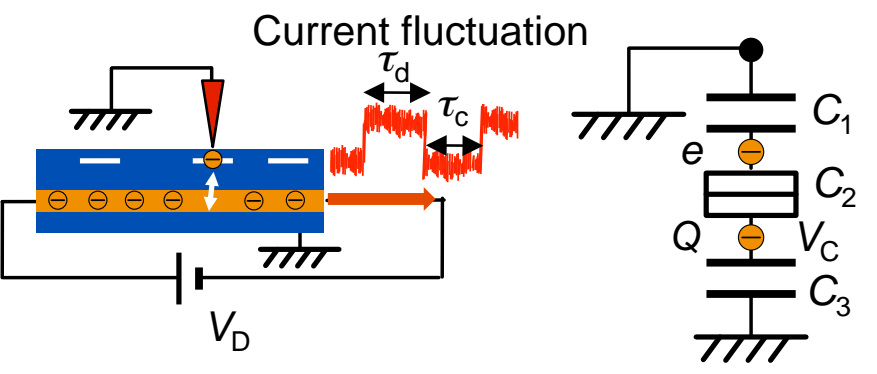

Fig. 1 Masaki Sato et al. 


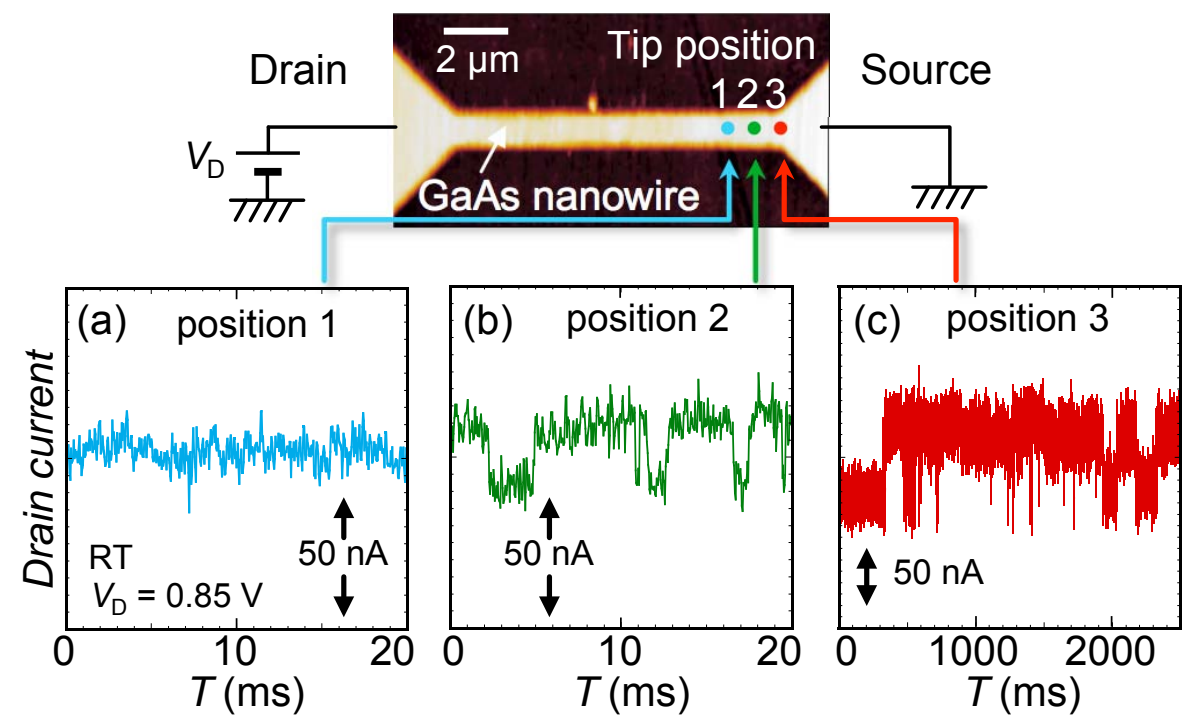

Fig. 2 Masaki Sato et al. 


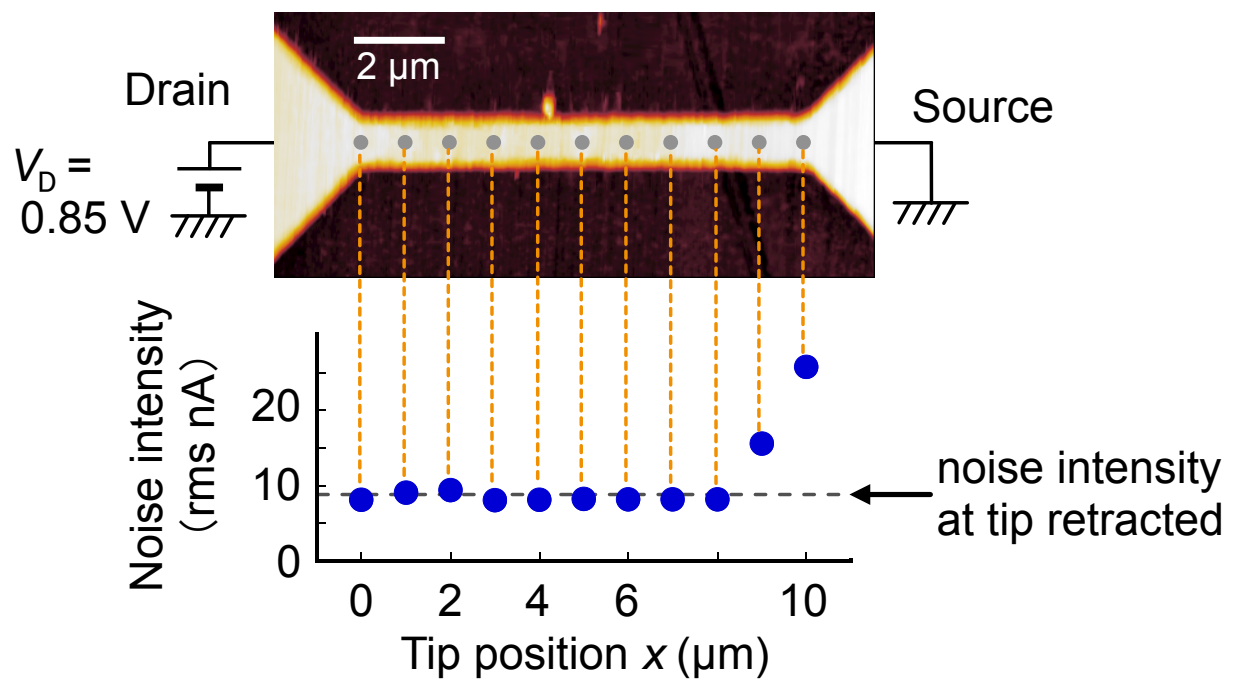

Fig. 3 Masaki Sato et al. 

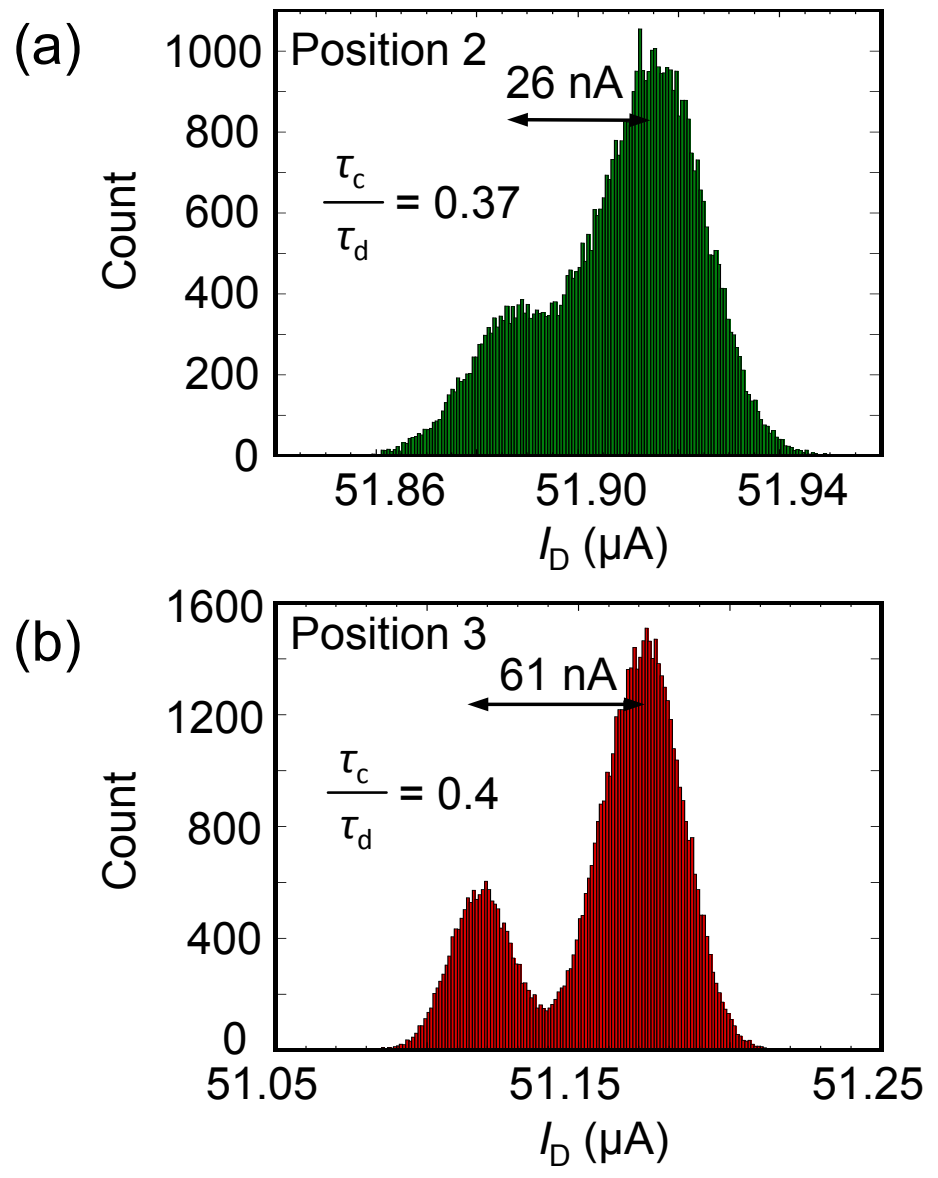

Fig. 4 Masaki Sato et al. 
(a)

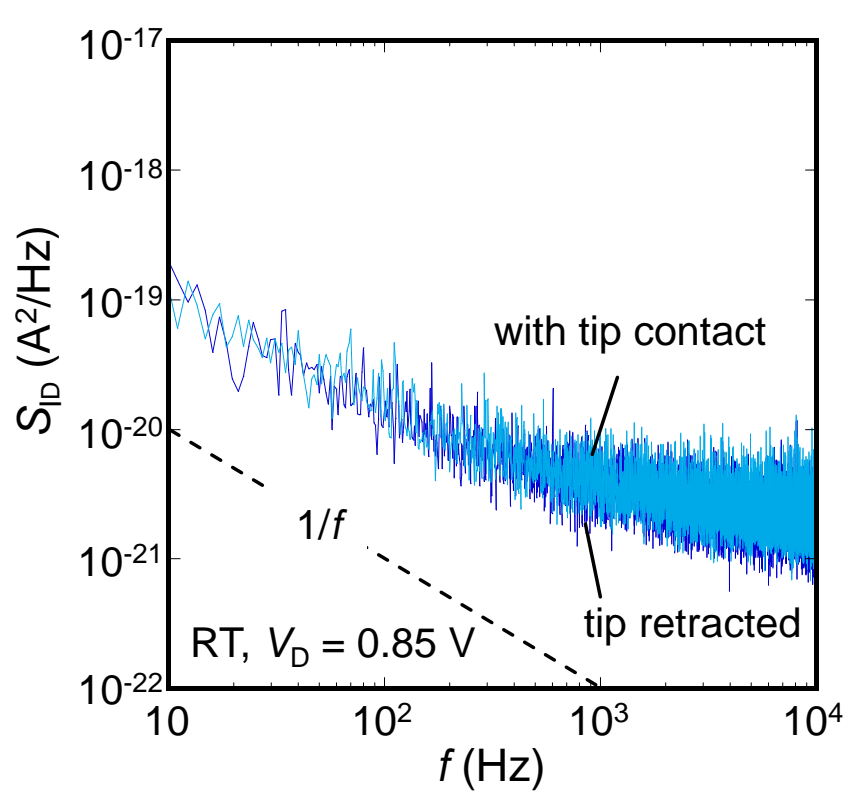

(b)

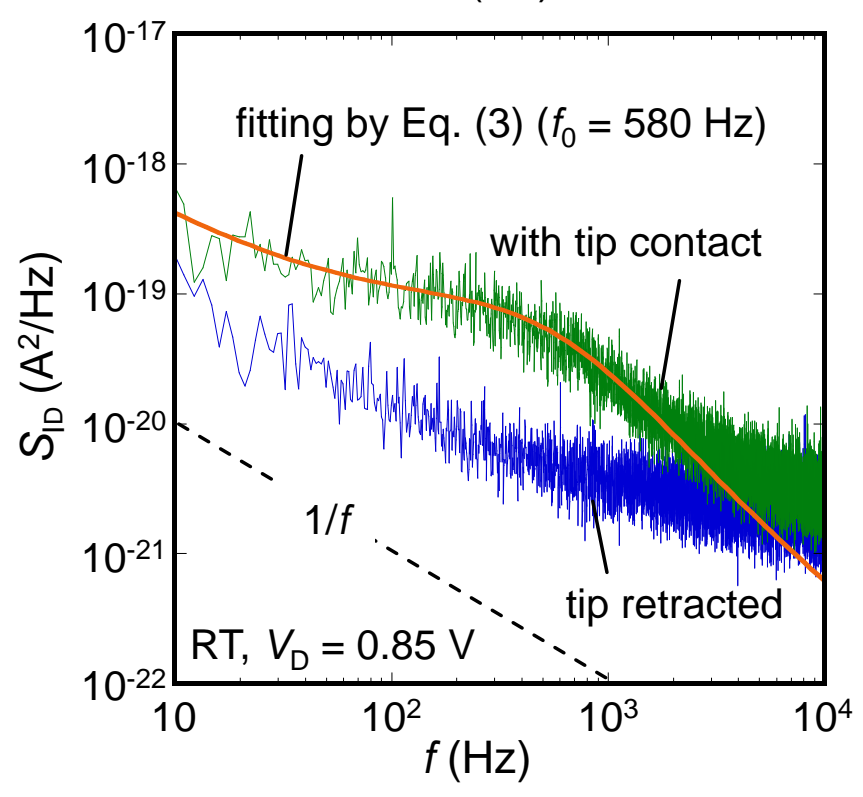

(c)

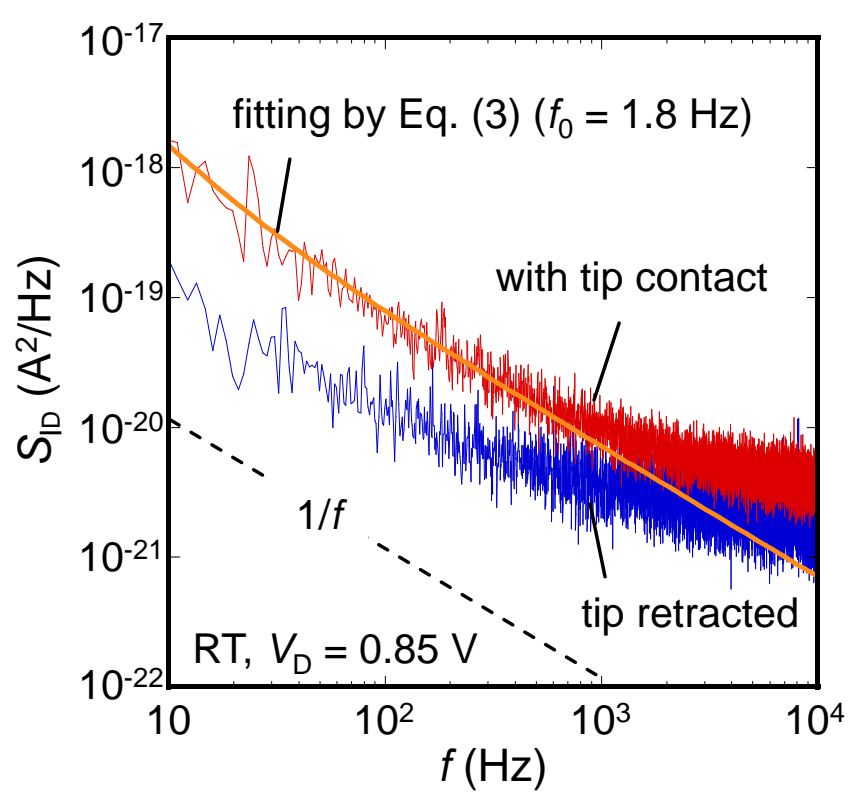

Fig. 5 Masaki Sato et al. 
(a)

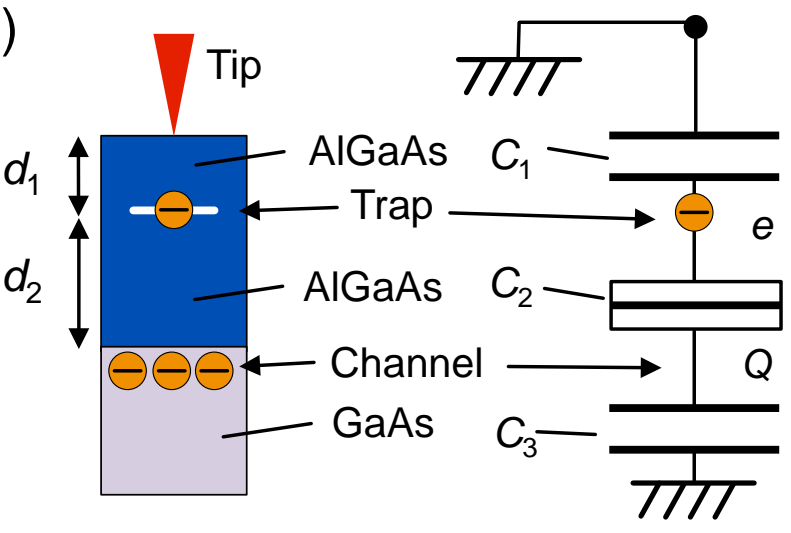

(b)

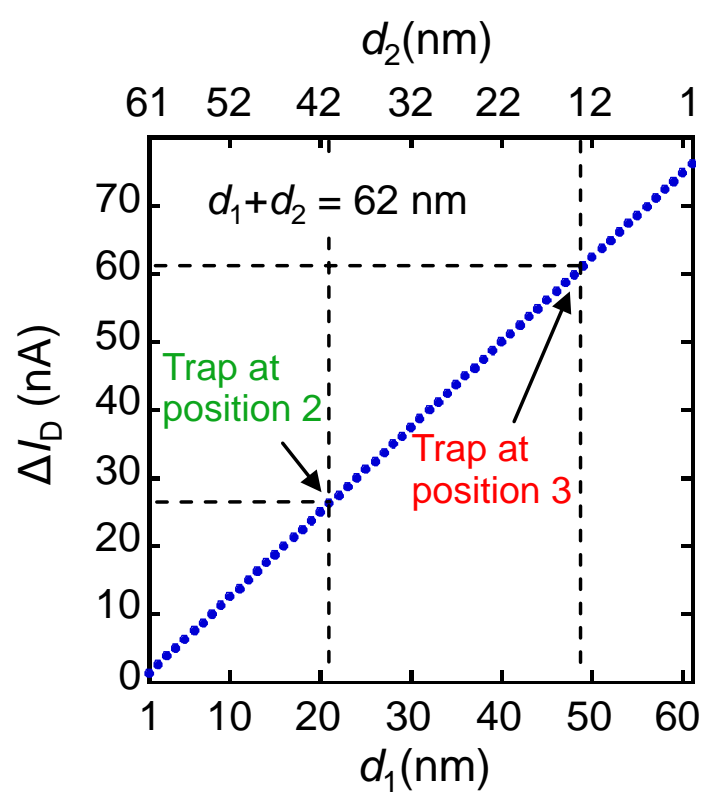

Fig .6 Masaki Sato et al. 